\title{
DENSIDAD OLIGODENDROGLIAL EN NERVIO ÓPTICO MURINO POR CONSUMO CRÓNICO DE METANOL
}

\author{
OLIGODENDROGLIAL DENSITY IN MURINE OPTIC NERVE \\ BY CHRONIC CONSUMPTION OF METHANOL
}

\author{
JOSÉ RAFAEL DÍAZ RUDAS \\ Universidad Norbert Wiener \\ PALMER HERNÁNDEZ \\ Universidad Norbert Wiener \\ MARÍA DE LOS ÁNGELES RIVERA \\ Universidad Norbert Wiener
}

\section{RESUMEN}

La mayoría de bebidas alcohólicas que se consumen en el Perú no cuentan con un registro sanitario; además, presentan componentes diferentes de los previstos en la norma. La adulteración de estas bebidas alcohólicas se evidencia en los niveles altos de etanol y bajos de metanol. El consumo agudo de concentraciones altas de metanol produce daño del nervio óptico y disminución de mielina y oligodendrocitos. El objetivo del estudio fue determinar el efecto del consumo crónico de concentraciones bajas de metanol en los oligodendrocitos del nervio óptico. Se utilizaron 60 ratas albinas macho, Rattus norvegicus, cepa Holtzman, edad 8 semanas. Fueron distribuidas aleatoriamente en 5 grupos de 12 ratas albinas cada uno: 1 grupo blanco y 4 grupos experimentales. Se utilizaron bebidas alcohólicas adulteradas: $\operatorname{pisco}(p)$, anisado $(a)$ y cognac $(c)$, y bebidas alcohólicas autorizadas: Pisco $(P)$ y Cerveza $(C)$ para elaborar los preparados alcohólicos correspondientes (grupos experimentales): $p a c, p a c+C$, Pisco y Pisco $+C$. La concentración de metanol en las bebidas alcohólicas adulteradas fue de 14,1 mg/100 ml (valor límite 150 mg/100 ml para bebidas destiladas según la Norma Técnica Peruana-Indecopi). El grupo blanco recibió agua ad libitum, y los grupos experimentales, una dosis diaria de 5,06 gramos de etanol $/ \mathrm{kg}$ de peso corporal por sonda orogástrica. Luego de 12 semanas las ratas albinas fueron sacrificadas por corte medular a nivel cervical; se extrajo y fijó el nervio óptico en formol neutro al $10 \%$. Posteriormente, se realizó un corte transversal a nivel proximal del nervio óptico y se procedió al estudio histopatológico de los oligodendrocitos, axón y mielina con la coloración de Hematoxilina \& Eosina. Se estudió la densidad oligodendroglial con el método estereológico del point counting, no encontrándose una diferencia significativa entre los 5 grupos mediante la prueba HDS Tukey $(\mathrm{p}=0,290)$. No existió variación en la densidad oligodendroglial. Se concluye que el consumo crónico de metanol a bajas concentraciones no produce alteración en la densidad oligodendroglial.

Palabras clave: metanol, nervio óptico, oligodendrocito, estereología, bebida alcohólica adulterada. 


\section{ABSTRACT}

Consumption of alcoholic beverages in Peru do not have a sanitary registry presenting components different from the regulations. fte adulteration of these alcoholic beverages is evidenced in high levels of ethanol and very low levels of methanol. Acute consumption of high concentration of methanol causes optic nerve damage with myelin and oligodendrocytes loss. fte purpose of this study was to investigate the effect of chronic consumption of low concentration of methanol on optic nerve oligodendrocytes. 60 male Holtzman rats, Rattus norvegicus, 8 weeks of age were used. Rats were randomly divided into five groups ( 1 control and 4 experimental). We used adulterated alcoholic beverages: $\operatorname{pisco}(p)$, aniseed $(a)$ and cognac $(c)$ and licensed alcoholic beverages: Pisco $(P)$ and Beer $(C)$ to prepare the corresponding alcoholic mixed drinks (experimental groups): $p a c, p a c+C, P i s c o$, $\mathrm{Pisco}+\mathrm{C}$. fte concentration of methanol in the adulterated alcoholic beverages was $14.1 \mathrm{mg} / 100 \mathrm{ml}$ (limit value $150 \mathrm{mg} / 100 \mathrm{ml}$ for distilled beverages according to the Peruvian Technical Standard-Indecopi). fte control group received water ad libitum and the experimental groups a dose of $5.06 \mathrm{~g}$ of ethanol $/ \mathrm{kg}$ body weight by orogastric tube. Twelve weeks later, the extraction of the optic nerve was carried out, fixing it in neutral buffer formalin $10 \%$ and then performing a transverse section at the proximal level of the optic nerve. Subsequently, a transversal section was made at the proximal level of the optic nerve and histopathological study of oligodendrocytes, axon and myelin with Hematoxylin \& Eosin staining was carried out. Oligodendroglial density was studied with the stereological method of point counting, and no significant difference was found between the 5 groups using the Tukey HDS test $(\mathrm{p}=0.290)$. Although there was no alteration in the oligodendroglial density. It is concluded that methanol at low concentration does not produce alteration in oligodendroglial density.

Key words: methanol, optic nerve, oligodendrocyte, stereology, adulterated alcoholic beverage.

\section{INTRODUCCIÓN}

El nervio óptico, también llamado segundo nervio craneal, transmite la información visual desde la retina hasta el cerebro. Nace de las células ganglionares de la retina y termina en el quiasma óptico (origen aparente), para luego llamarse tracto óptico. Los axones de las células ganglionares de la retina están mielinizados por los oligodendrocitos y se sitúan por fuera del globo ocular; estos axones forman el nervio óptico. El nervio óptico está rodeado por meninges a lo largo de todo su recorrido. Se origina a partir del tallo óptico en la semana siete del desarrollo embrionario y está compuesto por los axones de las células ganglionares de la retina y células gliales $^{1,2}$. La historia de la numeración de los nervios se remonta a la clasificación de Galeno en 7 pares (siglo II d. C.), para pasar luego a los 9 pares de Willis (1664) y, finalmente, a la versión moderna de los 12 pares de Soemmerring (1778). Sin embargo, desde finales del siglo XIX se generaun debate sobre el verdadero origen del nervio óptico. Se ha reconocido que el segundo par craneal es diferente de los oncepares craneales restantes, y debe considerarse parte del sistema nervioso central (SNC) porposeeroligodendrocitos y nocélulas de Schwann, como el sistema nervioso perifé$\operatorname{rico}(\mathrm{SNP})^{3,4,5}$. 
Existen dos tipos deoligodendrocitos, también llamados oligodendroglías: los oligodendrocitos interfasciculares -que se encargan de la producción de la vainade mielina y aislamiento del axón-y los oligodendrocitos satelitales, de los cuales aún no se precisa su función. Desde nuestro conocimiento sobreel oligodendrocitohumanolabiologíaes limitada; los resultados de estudios de roedores son a menudo extrapolados a los humanos. El gran número de oligodendrocitos en humanos es exponencialmente elevado, aunque la densidad de oligodendrocitos por mm3 es notablemente similar entre roedores y humanos ${ }^{6}$ (véase Figura 1).
Los oligodendrocitos son células posmitóticasque se desarrollan de células precursoras de oligodendrocitos (OPC), que migran dentro de la sustancia blanca en desarrollo desde sus zonas germinales. El número final de oligodendrocitos en cualquier parte del SNC podría, en principio, depender de la cantidad de OPC que migran a él, la cantidad de veces que los OPC se dividen antes de que se diferencien, y la cantidad de oligodendrocitos y OPC que experimentan muerte celular normal en la región $^{7,8,9}$.

Bebida alcohólica adulterada es aquella que ha sido privada parcial o totalmente de

\section{FIGURA 1}

Oligodendrocitos. Los oligodendrocitos son más pequeños y tienen menos prolongaciones que los astrocitos. Cada oligodendrocito envía varias prolongaciones que se enrollan en los axones, formando entre 30 a 100 capas concéntricas alrededor del mismo. Un oligodendrocito puede mielinizar de 30 a 50 axones adyacentes.
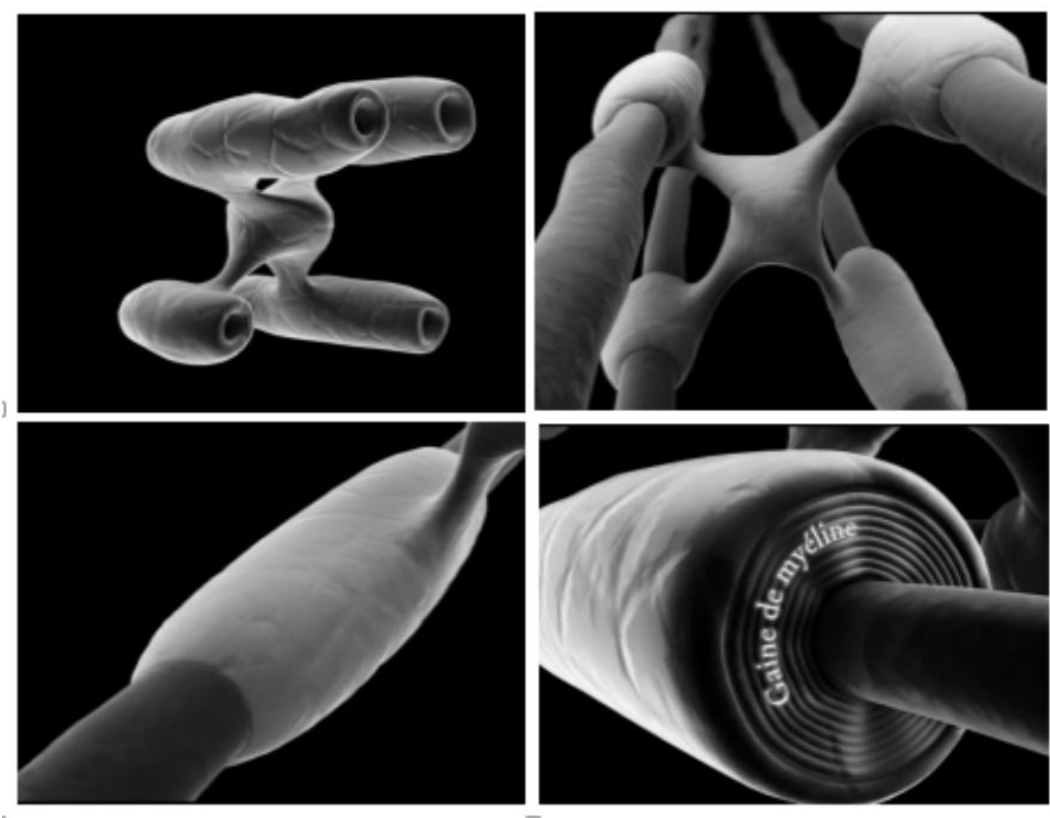

Fuente: http://neuromatiq.com/fr/chapitres/3-principes-de-base/03-les-cellules-gliales 
sus elementos característicos, reemplazándoselos o no por otros inertes o extraños de cualquier naturaleza para disimular $\mathrm{u}$ ocultar alteraciones, deficiente calidad de materias primas, defectos de elaboración, o para modificar la medida del producto. La produccióninformal e ilícita de bebidas alcohólicas se practica en muchas partes del mundo, incluso en países donde el alcohol está prohibido. Algunos nombres comunes para estas bebidas son moonshine (EUA), kumi kumi (Kenia), tuak (Malasia), tonayán (México), racumín, tumbaloco y penal en el Perú. Los consumidores eligen estas bebidas alcohólicas adulteradas debido a su bajo costo ${ }^{10,11}$.

El metanol o alcohol metílico es un productodelafermentación, especialmente cuando los mostos de uva sonmacerados con las cáscaras, y se encuentra tanto en bebidas fermentadas alcohólicas como no alcohólicas. El metanol aparece en toda la fase de destilación del pisco, en mayor proporción en la primera parte o cabeza; la cabeza es la primera porción de destilado que sale de los alambiques, cuyo grado alcohólico es alto. La única razón por la que un pisco podría presentar niveles de metanol por encima de la norma es porque hasidomalelaboradoo poradulteración ${ }^{12}$.

Entre las propiedades físicas del metanol destacan su densidad de $792 \mathrm{~kg} / \mathrm{m} 3$ y punto de ebullición de $64,7^{\circ} \mathrm{C}$, por lo que se evapora antes que el alcohol etíli- co o etanol, y se encuentra en cantidades importantes en la cabeza de los destilados, pero también en la cola, el último chorro, con un nivel muy bajo de alcohol; ambos son desechados en la destilación. La dosis letal mínima oral en humanos es de 0,3-1 $\mathrm{g} / \mathrm{kg}$ de peso corporal, y dosis mayores a $1,4 \mathrm{~g} / \mathrm{kg}$ causan la muerte. La ingestión de 10-30 ml (dosis tóxica) de metanol puro en adultos puede causar daño permanente en el nervio óptico, mientras que el coma y la muerte ocurren con volúmenes de 30-240 ml (dosis letal). El metanol, cuando se encuentra en concentraciones ligeramente superiores a las permitidas, se percibe irritante en boca y nariz, con matices intensos de olor. De acuerdo al Reglamento de la Denominación de Origen Pisco $^{13}$, la concentración de metanol deberá encontrarse en los rangos de 4-150 $\mathrm{mg} / 100 \mathrm{ml}^{14,15,16}$.

El etanol es absorbido en el estómago e intestino delgado. Su metabolismo está controlado en gran parte por el hígado, cuyos hepatocitos contienen la enzima alcohol deshidrogenasa $(\mathrm{ADH})$ y oxidan el etanolen acetaldehído. Luego, este es oxidado a ácido acético dentro de la mitocondria delhepatocito. Existe una segunda vía menos conocidalocalizadaen el cerebro:elcomplejo enzimático Microsomal Ethanol Oxidizing System (MEOS), que presentacitocromos, los cuales soninducidos por el etanol-igual como sucede en el hígado-. El metanol es absorbido y rápidamente distribuido por el agua del cuerpo, no se une a proteínas y su vida media oscila entre 2 y 24 horas. De modo similar al etanol, el metanol es metabolizado principalmente en la mitocondria delhepatocitoen el hígado.LaenzimaADH looxidaa formaldehídoy subsecuentemente por el aldehído-deshidrogenasa a ácido fórmico. Tanto el etanol como el metanol compiten por la enzima $\mathrm{ADH}$, aunque esta enzima prefiere metabolizar el etanol (afinidad 20 veces mayor); por ello, el tratamiento para la intoxicación por metanol se basa en el uso de etanol y hemodiálisis del metabolito (ácido fórmico) en casos graves ${ }^{17-21}$.

Los estudios sobre intoxicación por metanol corresponden a un consumo agudo, en un solo momento, y tratan respecto a las 
lesiones producidas sobre todo en el tejido nervioso debido a las altas concentraciones que presentarían algunas bebidas alcohólicas adulteradas, suceso que ocurre esporádicamente en el Perú. Poco se ha estudiado sobre intoxicación crónica con metanol a concentraciones bajas. El objetivo de este estudio fue determinar el efecto del consumo crónico de concentraciones bajas de metanol -presente en las bebidas alcohólicas adulteradas- en los oligodendrocitos del nervio óptico de rata albina, para así extrapolar los hallazgos a humanos.

\section{MATERIALES YMÉTODOS}

\section{MATERIALES}

\section{Muestra}

Se utilizaron 60 ratas albinas, Rattus norvegicus, cepaHoltzman. Edad: 8 semanas, sexo masculino, $120 \pm 20$ gramos de peso corporal. Lugar de manutención y procedimientos: bioterio animal de la Escuela de Medicina Humana de la Universidad Norbert Wiener (UNW); alimentación con cebada (10 g de cebada/100 g de peso corporal) y agua ad libitum. Cada rata albina fue colocada en una jaula funcional de malla metálica de 12 litros de volumen $(30 \mathrm{~cm}$ de profundidad x $20 \mathrm{~cm}$ de alto x $20 \mathrm{~cm}$ de ancho). El bioterio se mantuvo con 12 horas de luz/oscuridad y temperatura de $24 \pm 2{ }^{\circ} \mathrm{C}$.

\section{Bebidas alcohólicas}

Secompróbotellas de piscoadulterado, cognac adulterado y anisado adulterado. Fueron obtenidas en el centro de la ciudad de Lima, a un costo de 22 soles por las 3 botellas. Asimismo, se compró botellas de pisco de venta autorizada, el más barato del mercado, y botellas de cerveza marca Pilsen. Se rotuló Pisco-en mayúsculas-a la bebida autorizada ( $P$ es su abreviatura) y pisco - en minúsculas-a la bebida adulterada ( $p$ es su abreviatura) para poder diferenciarlas; asimismo, Cerveza-con mayúsculas-a la bebida autorizada ( $C$ es su abreviatura).

\section{Sonda orogástrica}

Para la administración diaria de las bebidas alcohólicas se confeccionó una sonda de $12 \mathrm{~cm}$ de largo a partir de una aguja de punción lumbar n. ${ }^{\circ} 20$ tipo Quincke, a la cual se le dio una curvatura y se le retiró el bisel;en su lugarse colocóunaesferahueca y roma de aleación de plata y bronce.

\section{MÉTODOS}

Preparados alcohólicos. A partir de las bebidas alcohólicas mencionadas se hicieron los siguientes preparados:

- "Tumbaloco", alquellamamos pac:partes iguales de pisco adulterado + anisado adulterado + cognacadulterado.

- "Penal", al que llamamos $p a c+C$ : partes iguales de pisco adulterado + anisado adulterado + cognac adulterado + cerveza Pilsen. - "Pisco", al que llamamos Pisco: el pisco de venta autorizada más barato del mercado.

- "Pisco+Cerveza", alque llamamos Pisco+C: tres partes del pisco de venta autorizada más barato del mercado + una parte de cerveza autorizada.

Aleatorización y distribución de la muestra.La muestrafuedistribuidaal azar en 5 grupos (1 grupo blanco y 4 grupos experimentales) de 12 ratas albinas cadauno, a las cuales se les administró de manera diaria y durante 12 semanas: agua ad libitum (grupo blanco) y las bebidas alcohólicas anteriormente mencionadas (grupos: pac, pac $+c$, Pisco y Pisco $+C$ ). 
Análisis cuantitativo de etanol y metanol en las bebidas alcohólicas adulteradas y autorizadas. Fue realizado en el Centro de Información, Control Toxicológico y Apoyo a la Gestión Ambiental (Cicotox) de la UNMSM, siguiendo el método de espectrofotometría UV-visible para límites de detección de $0,02 \mathrm{mg} / \mathrm{ml}$ y cromatografía de gases para límites de detección de $0,01 \mathrm{mg} / \mathrm{ml}$.

Cálculodeladosis.Sesiguióel cálculo realizado por Díaz ${ }^{22}$. Al grupo experimental sele administró una dosis estandarizada de etanol obtenida por extrapolación de una persona adulta de sexo masculino de $70 \mathrm{~kg}$ de peso corporal que consumauna botella diaria $(750 \mathrm{ml})$ de bebida alcohólica adulterada al $15 \%$ de etanol durante un periodo mayor de 5 años.

Una de las mayores confusiones al administrar una dosis adecuada de etanol en ratas albinas es la ligereza con la que se revisan las condiciones experimentales al efectuar comparaciones entre diferentes estudios. El metabolismo basal, es decir, la producción de calor durante 24 horas en condiciones de reposo, al ser calculado por kilogramo de peso corporal resultó mayor mientras menor fuera el peso corporal del animal a estudiar. Según la medición del metabolismoo ley dela superficie corporal de Voit, la cantidad de Kcal/Kg/día en el hombre es de 32,1; para la rata albina es de 128,4. Por tanto, el metabolismo basal para la rata albina es aproximadamente 4 veces mayor que en el hombre, por lo que se les administró diariamente a las ratas una dosis multiplicada por el factor 4 , en comparación con el serhumano.

El grupo experimental recibió una dosis de 5,06 gramos de etanol/kg de peso corporal. La LD50 para consumo oral de etanol en ratas albinas es de 7,06 g/ $\mathrm{kgde}$ peso corporal; la dosis diaria aplicada fue inferior a esta. El volumen administrado fue de $4 \pm 2 \mathrm{ml}$; el volumen máximo del estómago de una rata albina adulta es de 7 $\mathrm{ml}$; el volumen administrado fue inferior a este. La LD50 para consumo oral demetanol en ratas albinas es de 5,628 g/kg de peso corporal; la dosis diaria administrada fue mucho menor a esta.

Manipulacióndela rataalbina y sondeo. Se sujetó suavemente a la rata albina, con la manoizquierda, colocando la palma sobre el dorso del animal de experimentación, con el dedo índice y medio alrededor del cuello, y los demás alrededor del tórax. Una vez inmovilizada, con la mano derecha se procedió al deslizamiento de la sonda orogástrica por la boca y esófago hasta llegar a la luz del estómago. Luego, con el dedo índice, se presionó lentamente el émbolo de la jeringa para administrar el volumen de bebida alcohólica asignado.

Sacrificio del animal de investigación. El sacrificio fue por un corte medular a nivel cervical; previamente, la rata albina fue anestesiada con la bebida alcohólica a la cual fue sometida de manera crónica durante el estudio.

\section{Extracción del nervio óptico y globo} ocular. Se observó si el nervio óptico seguía aún unido al quiasma óptico; en caso estuvieran unidos, se les separómediante un corte transversal con hoja de bisturí $n$. $^{\circ}$ 11. Estando el nervio óptico unido solo al globo ocular y a la piel, músculo yhueso que lo rodean, se procedió a cortar la piel restante, y, con tijera recta, el hueso maxilar en sentido recto hacia la nariz. El corte se realizó teniendo como soporte una sonda acanalada, la cual se atravesó por encima del globo ocular; luego se fragmentó 
el hueso que estuvo alrededor del globo ocular con una pinza hemostática. Esta acción se realizó con precisión, pues aquí se encuentra el agujero por donde transcurre el nervio óptico; luego se removió la parte ósea con pinza plana. Se procedió a extraer el nervio óptico junto a otros pares craneales (pares óculomotores y nervio trigémino) (véase Figura 2). Con una pinza recta se cogió el nervio óptico desde su inicio en el globo ocular y se cortaron los músculos que los mantenían unidos. Luego se colocó el nervio óptico y globo ocular en un fragmento de depresor lingual de madera (bajalenguas), de dimensiones $2 \mathrm{~cm}$ de ancho x $3 \mathrm{~cm}$ de largo x $1 \mathrm{~mm}$ de ancho, mediante hilo de seda negra, atando en un extremo el globo ocular y en el otro la parte distal del nervio óptico. Se estiró suavemente la muestra extraída y se le anudó al depresor lingual. Posteriormente se colocó el depresor lingual en un frasco de formol neutro al $10 \%$, de manera que la cara que contenía al nervio óptico quedara en contacto directo con el formol.

\section{Separación nervioóptico-globo ocu-} lar. Una semana después del proceso de fijación en formol se realizó, mediante corte transversal, la separación del nervioóptico del globo ocular en su porción rostral; el nervio fue colocado en forma perpendicular al molde de base metálica para su inclusión posterior en parafina.

Deshidratación, aclaramiento e inclusión. Este proceso se llevó a cabo en el Departamento de Neuropatología del Instituto Nacional de Ciencias Neurológicas (INCN). Se deshidrató la muestra utilizando soluciones de alcohol en orden creciente. Para el aclaramiento se utilizó butoxietano en lugar de xilol. Posteriormente se colocó la muestra en parafina para proceder a realizar cortes transversales de nervio óptico de $8 \mu \mathrm{m}$ de espesor. Se montaron

\section{FIGURA 2}

Encéfalo murino. Esquema del encéfalo de nervio óptico en vista lateral izquierda mostrando ambos nervios ópticos (NO).

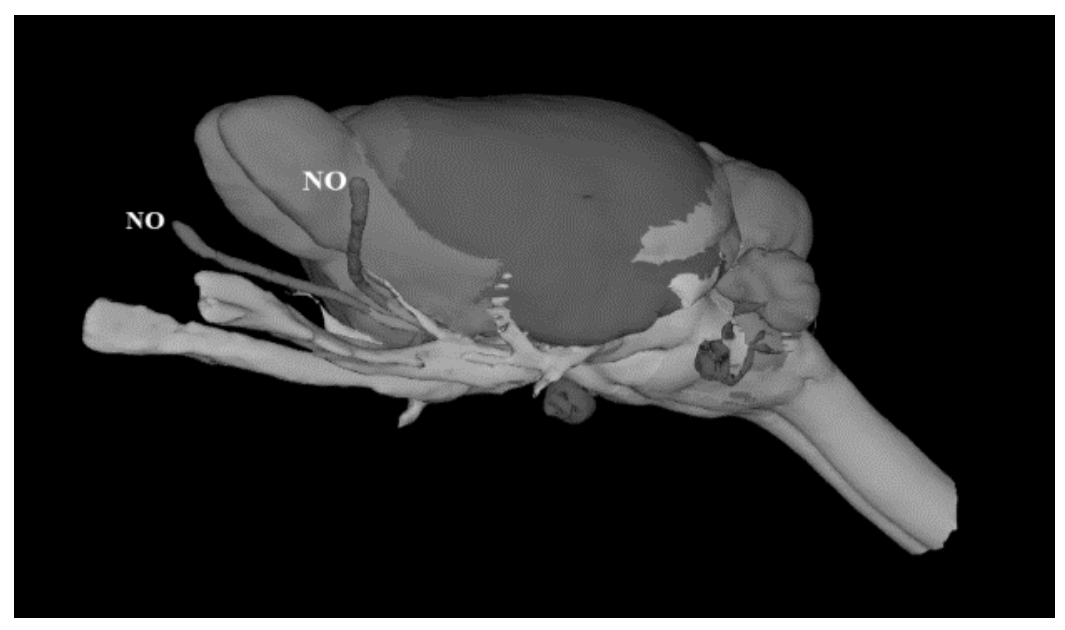

Fuente: fte Waxholm Space Sprague Dawley rat atlas of Papp et al. (2014). 
los cortes en láminas y se empleólatinción Hematoxilina \& Eosina (H\&E).

Micrografiado a 400x. Se utilizó un microscopiobiológicoEclipseCi-LNikon con una cámara adaptada al fototubo del mismo. Tres campos de nervio óptico fueron estudiados por cada rata albina.

Morfometría, estereología. La estereología es el conjunto de métodos con el cual se obtienen datos cuantitativos tridimensionalesa partirde secciones bidimensionales. Se trata de una extrapolación del plano al espacio, de acuerdo con el resultado de diferentes conceptos teóricos de estadística, geometría integral y probabilidad geométrica. Es importante resaltar que es una herramienta libre de sesgo, pues presenta medidas de cuantificación aprovechando propiedades geométricas intrínsecas de la estructura a estudiar: tamaño, forma, tipología y función.

Se realizó en primer lugar el estudio morfométricoidentificando la morfología o citoarquitectura de los oligodendrocitos, para confirmar que el campo de estudio correspondieraal nervioópticoy no al nervio periférico (aquí se encuentran las células de Schwann). Ya identificados los oligodendrocitos, se procedió a diferenciarlos de los astrocitos y células ganglionares.

Para realizar la morfometría de las láminas de nervio óptico en corte transversal se utilizó el software JMicroVision versión 1.2.7; se evaluó la densidad de los oligodendrocitos por campo con el método del point counting de acuerdo a la siguiente fórmula: número de puntos que toca a un oligodendrocitomultiplicado porel número de puntos estudiados y dividido todo entre el número de puntos totales del campo (llámese número de puntos estudiados a aquellos puntos que excluyen laspartes que estén vacías en el número de puntos totales de campo). El número de puntos totales que existen en nuestra cuadrícula y se superponen a un campo de 400x es de 70: 10 puntos a lo largo y 7 puntos a lo ancho.

Estadística. Se empleó el software IBM SPSS Statistics versión 22 y se realizó un análisis descriptivo utilizando el estadístico promedio. Se utilizó la prueba de Kruskal Wallis para comparar la media aritmética entre los 5 grupos (grupo blanco y grupos experimentales), pues los datos presentaron una distribución no normal. Parala pruebadecomparaciones múltiples se utilizó la prueba HSD de Tukey.

Ética. Se siguieron las normas establecidas en la Ley 30407, Ley de protección y bienestaranimal,queestipulalascondiciones éticas parael manejo de animales de laboratorio. Se rechaza así cualquier acto de crueldad y se evita, de la misma manera, sufrimiento innecesario para el animal de laboratorio ${ }^{23}$.

\section{RESULTADOS}

En contraste con lo hallado por Díaz $2015^{17}$, en los últimos 13 años, periodo 2004-2017, la concentración de etanol ha aumentado en 3 veces, mientras que la concentración de metanol ha disminuido hasta en 150 veces. El valor límite de metanol es de alrededor de $300 \mathrm{mg} / 100 \mathrm{ml}$ (o su equivalente en $3000 \mathrm{mg} / \mathrm{lo} 3 \mathrm{~g} / \mathrm{l}$ ), según las distintasnormasinternacionales, yes de $150 \mathrm{mg} / 100 \mathrm{ml}$ según la NTP-Indecopi. El promedio de metanol en las bebidas alcohólicas adulteradas del estudio fue de $14,13 \mathrm{mg} / 100 \mathrm{ml}$, mientras que el promedio en las bebidas alcohólicas autorizadas del estudio fue menor a $0,01 \mathrm{mg} / 100 \mathrm{ml}$. 
Esto se contrapone a la cantidad de metanol hallada en 2004, que fue 15 veces superior a la NTP22 (véase Tablas 1 y 2).

El tamaño de nervio óptico en las ratas albinas es de $20 \mathrm{~mm}$; el humano es de 50 $\mathrm{mm}$. Se logró una buena técnica de exten- dido del nervio óptico al colocarlo junto al paquete muscular en un fragmento del depresor lingual de madera, lo que evitó su retracción y espiralización. Ello facilitó realizar los cortes transversales. Al microscopio óptico se evidenció y confirmó que la ubicación de la arteria central de la reti-

TABLA 1

Bebidas alcohólicas. Concentración de metanol y etanol.

\begin{tabular}{lllll}
\hline \multirow{2}{*}{$\begin{array}{c}\text { Bebidas } \\
\text { alcohólicas }\end{array}$} & \multicolumn{2}{c}{ Metanol (mg/100 ml) } & \multicolumn{1}{c}{ Etanol (ml/100 ml) } \\
\hline \multirow{2}{*}{$p=$ pisco adulterado } & Año 2004 & Año 2017 & Año 2004 & Año 2017 \\
\cline { 2 - 5 }$a=$ anisado adulterado & 1967 & 10,0 & 23,68 & 33,00 \\
$c=$ cognac adulterado & 1725 & 17,4 & 14,16 & 23,60 \\
$P=$ Pisco autorizado & no estudiado & menor a 1 & no estudiado & 42,00 \\
$C=$ Cerveza autorizada & no estudiado & menor a 1 & no estudiado & 5,00 \\
\hline
\end{tabular}

TABLA 2

Preparados alcohólicos. Concentración de metanol y etanol.

\begin{tabular}{lllll}
\hline $\begin{array}{l}\text { Preparados } \\
\text { alcohólicos }\end{array}$ & Metanol (mg/100 ml) & \multicolumn{2}{c}{ Etanol (ml/100 ml) } \\
\hline \multirow{2}{*}{ pac } & Año 2004 & Año 2017 & Año 2004 & Año 2017 \\
\cline { 2 - 5 } pac $+C$ & 2210 & 14,13 & 16,90 & 30,70 \\
$P i s c o$ & 1658 & 10,60 & 13,90 & 24,20 \\
Pisco $+C$ & no estudiado & menor a 1 & no estudiado & 40,00 \\
& no estudiado & menor a 1 & no estudiado & 31,20 \\
\hline
\end{tabular}

na murina es por fuera del nervio óptico, a diferencia del humano que discurre por el centro del nervio óptico (véase Figura3). El nervio óptico presentó una fina cubierta demeninges, adiferenciadelnervioperiférico, que está cubierto de epineuro. El color del nervio óptico en H\&E es de un tono más violetaqueelnervio periférico, lo cual ayudó a diferenciarlos de manera rápida. Revista de Investigación de la Universidad Norbert Wiener, 2018, N. ${ }^{\circ} 7$
En el estudio morfométrico se observa que el tamaño del oligodendrocito es el mismo en todos los grupos; no hubo alteración en su morfología nuclear, ligeramente ovalada, ni en sus prolongaciones citoplasmáticas (no evidenciadas en condiciones normales en coloración H\&E al microscopio óptico). El diámetro mayor fue de $5 \mu \mathrm{m}$, y el diámetro menor, de 3 
FIGURA 3

Nervio óptico. Corte transversal.

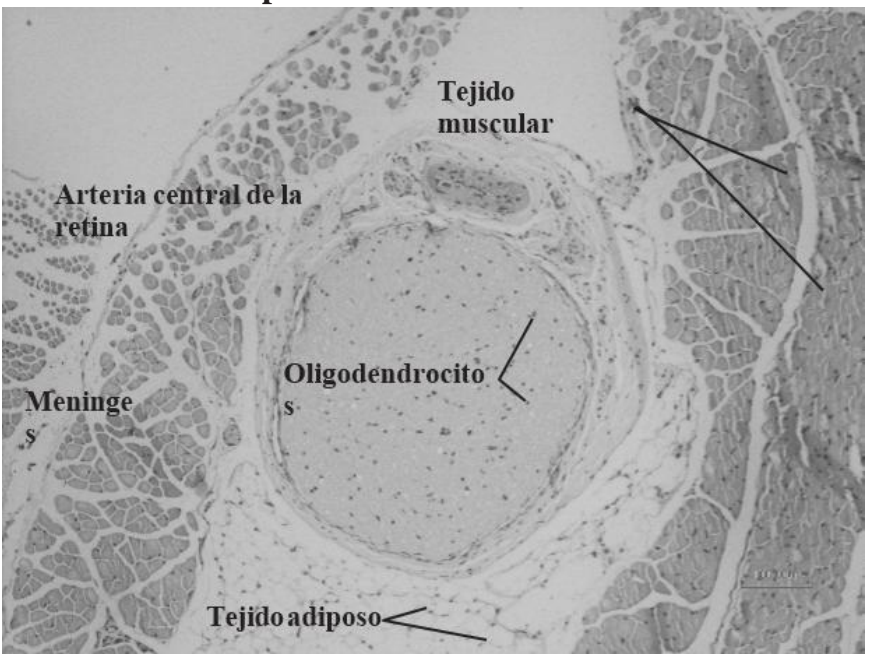

$\mu \mathrm{m}$; presentaron una tinción nuclear eosinófila oscura y, en la mayoría de casos, rodeados de un halo blanco citoplasmático en forma de huevo frito. Asimismo, en un corte transversal del nervio óptico se encontró una distribución aleatoria de oligodendrocitos y no lineal, como en un corte longitudinal.

Respecto al debate sobre si el ner- vio óptico debería considerarse parte del SNC, nuestra propuesta es que sí: se presentó como glías formadoras de mielina a los oligodendrocitos y no alas células de Schwann. La distribución aleatoria de oligodendrocitos fue la misma que el SNC. Incluso, de no saber que el tejido era el nervio óptico, podríamos haber afirmado que estábamos en sustancia blanca de SNC. Explicado esto, se comprendió por qué el globo ocular es considerado una prolongación del cerebro.
No existe atrofia ni disminución de superficie del nervio óptico en su corte transversal que enmascare la pérdida de oligodendrocitos; el nervio óptico presentó una estructura homogénea y, así mismo, una distribución aleatoria de los oligodendrocitos. La estereología es el mejor método de conteo de la densidad oligodendroglial, pues no se contaron todos los oligodendrocitos existentes en un campo de 400x, sino que se utilizó el método del point counting mediante una cuadrícula colocada al azar que nos permitió contar solo los oligodendrocitos tocados por los puntos de conteo (intersección de líneas).

Para la densidad oligodendroglial se realizó la prueba de homogeneidad de varianzas; como resultado se observó una distribución no normal de los datos. Por tal motivo, y por tener 5 grupos para comparar, se utilizó la prueba no paramétrica de 
Kruskal Wallis, la cual nos indicó que no existen diferencias significativas $(\mathrm{p}=0,556)$ entre los 5 grupos. Se realizó el test HSD de Tukey, el cual confirmó que no existen diferencias significativas entre los grupos al compararlos entre sí (véase Tabla 3 ).

\section{DISCUSIÓN}

Para el análisis de la concentración de metanol en las bebidas alcohólicas adulteradas y autorizadas se seleccionóla norma técnica ecuatoriana de determinación de metanol, que tuvo éxito tanto en ese país como a nivel mundial debido a la innovación en la detección de metanol en bebidas alcohólicas adulteradas. Se utilizóel métodode análisis cuantitativo por espectrofotometría UV-visible ${ }^{24}$.

Sobre el origen de las bebidas alcohólicas adulteradas (pisco, anisado y cognac adulterados) se plantean tres posibilidades: se trataría de alcohol industrial, alcohol rectificado o fracciones desechadas de la destilación, es decir, cabeza y cola. No po- dría tratarse de alcohol industrial pues al ser este un subproducto del etanol tendría un alto contenido de aldehídos y ésteres, entre otros. Podría el origen del metanol de las bebidas alcohólicas adulteradas provenir de la cabeza, al ser el metanol uno de los alcoholes más livianos en el proceso de destilación. Al analizarel metanol yetanol de las bebidas alcohólicas adulteradas se encontró cantidades elevadas de etanol, pero muy bajas de metanol, incluso por debajo de los valores permitidos; tal es el caso del pisco adulterado, que contiene 10 $\mathrm{mg} / 100 \mathrm{ml}$, cuando la NTP establece márgenes comprendidos entre 4 y $150 \mathrm{mg} / 100$ $\mathrm{ml}$. El promedio encontrado en el pisco de venta autorizada fue de $65 \mathrm{mg} / 100 \mathrm{ml}$. Por lo antes expuesto, las concentraciones bajas de metanol no provendrían ni del alcohol industrial ni de las fracciones desechadas de la destilación, quedando abierta la posibilidad de tratarse de alcohol rectificado. Esto explicaría el por qué de la Ley $29632^{25}$. cuyo fin es desnaturalizar el alcohol rectificado y evitar así su utilización en la producción de bebidas alcohólicas.

TABLA 3

Pruebas de comparaciones múltiples

\begin{tabular}{lll}
\hline & \multicolumn{2}{c}{ Densidad oligodendroglial } \\
\cline { 2 - 3 } Bebidas alcohólicas & $\mathrm{N}$ & 1 \\
Pac & 12 & 2,12 \\
Pisco & 12 & 2,77 \\
Blanco & 12 & 2,89 \\
Pac + c & 12 & 3,44 \\
Pisco + C & 12 & 3,80 \\
\hline Significatividad & & 0,290
\end{tabular}

La prueba pos hoc de Tukey muestra que no existe diferencia significativa entre los 5 grupos. 
Las concentraciones bajas de metanol encontradas en las bebidas alcohólicas adulteradas están por debajo de la NTP; consiguientemente, no llegan al LD50 para consumo oral de etanol en ratas albinas. La neuropatía óptica por consumo crónico de concentraciones bajas de metanol podría ser acumulativa en el tiempo, pero a su vez el metabolismo basal de la rata albina es elevado y depuraría el metanol sistémico.

El point counting fue el método estereológico escogido para el estudio de la densidad oligodendroglial, pues el tamaño del oligodendrocito es mayor al punto de intersección de las líneas de la cuadrícula; por tanto, no hay sesgo en el conteo de los mismos.

Los oligodendrocitos no tienen capacidad regenerativa del nervio óptico, y por ello no pueden aumentar en número en el SNC. La alteración en el número de estos implicaría una injuria que perjudicaría el axón o su mielina; frente a esto, el número de oligodendrocitos aumentaría como mecanismo de defensa. Sin embargo, frente a una injuria en el SNClos axones lesionados no se regeneran debido a que los oligodendrocitos y la mielina liberan factores inhibidores de crecimiento axonal, tales como Nogo-A, glicoproteína asociada a mielina (MAG) y glicoproteína oligodendrocito de mielina $(\mathrm{MOG})^{26-30}$.

Pero, ¿qué se daña con el metanol exactamenteen el nervioóptico? ¿Laneuropatía óptica por metanol a bajas concentraciones daña el axón, la mielina o el oligodendrocito? El mecanismo del daño del nervio óptico por metanol no está del todo esclarecido. Estudios previos mencionan un daño primario de la retina (neuronas que dan origen al nervio óptico), mientras que otros estudios mencionan un daño primario del nervio óptico, con daño posterior de la retina. No hubo, en nuestro estudio, alteración alguna del número de axones, mielina u oligodendrocitos, por lo que es probable que el daño se observe, si lo hubiera, en la retina misma, en las células ganglionares.

Es posible que el nerviomorfológicamente esté intacto, llámese nervio al paquete que incluye axón, cubierta de mielina y oligodendrocito. Cabe recordar que los estudios de microscopíaóptica evalúan la morfología tisular y a partir de ello se infieren posibles alteraciones en la fisiología normal del tejido; por tanto, existe la posibilidadde una alteraciónfisiológicano cuantificada al momento del estudio por microscopía óptica o que el daño aún no se evidencie ysea inminente.

En una investigación previa, Díaz 2017, encontramos que el consumo crónico de pisco de venta autorizada más barato del mercado causa mayor densidad neuronal aponecrótica en la corteza piriforme. La pregunta ahora es ¿por qué no se produce aquel mismo daño en el nervio óptico? Posibles respuestas: los daños son distintos según los tejidos, la concentración de metanol administrada a la rata albina fue baja, o la morfología es normal, pero la fisiología está alterada.

El tiempo de administración de las bebidas alcohólicas en ratas albinas fue de 12 semanas, que corresponderían a siete años de consumo crónico en humano, tiempo suficiente para activar mecanismos compensatorios que eviten la aparición de la enfermedad. Probablemente, al inicio de la administración crónica de bebidas alcohólicas sí hubo daño morfológico cuantificable o tal vez se encontraría en un lapso mucho mayor de administración. Se trabajó estandarizandola misma dosis de etanol 
y no de metanol, pues las concentraciones de metanol se encontraron incluso $10 \mathrm{ve}$ ces por debajo de la NTP.

Nuestro estudio pretende replicar la realidad del consumo crónico del alcohol metílico, es decir, el consumo en bajas concentraciones durante un largo periodo; no se asemeja a los estudios que utilizan concentraciones elevadas de metanol para replicar intoxicaciones agudas.

Para estudios posteriores con metanol a bajas concentraciones en ratas albinas, se recomienda utilizar una larga data, mayor a doce semanas, y realizar estudios histopatológicos seriados; asimismo, estudiar la densidad de las neuronas ganglionares de la retina del globoocular.

\section{CONCLUSIÓN}

El consumo crónico de metanol a bajas concentraciones, presentes en las bebidas alcohólicas adulteradas, no produce alteración en la morfología ni en la densidad oligodendroglial.

\section{REFERENCIAS BIBLIOGRÁFICAS}

1. Reeves C, Taylor D. A history of theoptic nerve and its diseases. Eye 2004; 18:1096-1109.

2. Moore KL, Persaud TV. Embriología clínica, 9aedición. España: Elsevier; 2013.

3. Shaw JP. A history of the enumeration of the cranial nerves by European and British anatomists from the time of Galen to 1895 , with comments on nomenclature. Clinical Anatomy 1992 Jan; 5(6):466-484.

4. Monro TK. Optic Nerve as Part of the Central Nervous System. Journal of Anatomy and Physiology 1895; 30(1): 45-48.

5. Bradl M, Lassmann H. Oligodendrocytes: biology and pathology. Acta Neuropathol 2010; 119:37-53.

6. Peters A. Observations on the connexions between myelin sheaths and glial cells in the optic nerves of young rats. Journal of Anatomy 1964; 98(1):125-134.

7. Barres BA, Raff MC. Axonal Control of Oligodendrocyte Development. J Cell Biol 1999 dec; 147(6): 11231128.

8. Baumann N, Pham-Dinh D. Biolo- gy of oligodendrocyte and myelin in the mammalian central nervous system. Physiol Rev 2001 Apr; 81(2):871-927.

9. Schwab ME, Schnell L. Region-specific appearance of myelin constituents in the developing rat spinal cord. Journal of Neurocytology 1989 Apr; 18(2) 161-169.

10. Giovanetti, F. Methanol poisoning among travellers to Indonesia. Travel Medicine and Infectious Disease 2013; 11(3):190-193.

11. Gee P,Martin E. Toxic cocktail: methanol poisoning in a tourist to Indonesia. Emergency Medicine Australasia 2012; 24: 451-453.

12. International Programme on Chemical Safety. Environmental Health Criteria No 196: Methanol. Geneva: World Health Organization, 1997.

13. Norma Técnica Peruana 211.001:2006. Bebidas alcohólicas. Pisco. Requisitos. 7. aedición.

14. World Health Organisation. Methanol: health and safety guide. Geneva: World Health Organization; 1997.

15. Wood CA, Buller F. Poisoning by wood alcohol. JAMA 1904 oct; 43(14): 972-977. 
16. Kobro M. Methanol Poisoning. Acta Pharmacologica et Toxicologica 1946 Apr; 2: 95-108.

17. Díaz R. Histomorfometría de la capa granular orbitofrontal murina sometida a administración crónica de bebidas alcohólicas adulteradas. Lima: Universidad Nacional Mayor de San Marcos, 2015.

18. Zakhari S. Overview: ¿How Is Alcohol Metabolized by the Body? Alcohol Res Health 2006; 29 (4): 245-254.

19. Sadun A. Acquired mitochondrial impairment as a cause of optic nerve disease. Trans Am Ophthalmol Soc 1998; 96: 881-923.

20. Sharma M, et al. Methanol-Induced Optic Nerve Cupping. Arch Ophthalmol 1999; 117: 286.

21. Grzybowski A, et al. Toxic optic neuropathies: an updated review. Acta Ophthalmol 2015; 93: 402-410.

22. Díaz R. Aponecrosis de la corteza piriforme en ratas albinas sometidas a administración crónica de bebidas alcohólicas adulteradas. Revista de Investigación de la Universidad Norbert Wiener 2017; 6:25-40.

23. Ley 30407, Ley de protección y bienestar animal. (El Peruano, 8 de enero de 2016).
24. Norma Técnica Ecuatoriana. NTE INEN 0343: Bebidas alcohólicas. Determinación de los aldehídos, 2015.

25. Ley 29632, Ley para erradicar la elaboración y comercialización de bebidas alcohólicas informales, adulteradas o no aptas para el consumo humano (El Peruano, 17 de diciembre de 2010).

26. Pérez-Olvera O, Arellano S, Rodríguez H. Revisión de los métodos estereológicos y su aplicación en biología celular. Patología 2012; 50(2): 63-71.

27. Cassini A, Levinas L, Pringe H. Einstein y el efecto Compton. Sci stud 2013; 11 (1): 185-09.

28. Garner C, Lee E. Evaluation of methanol-induced retinotoxicity. Toxicology 1994 Jan; 93: 113-124.

29. Qui J, Cai D, Filbin MT. Glial inhibition of nerve regeneration in the mature mammalian CNS. Glia 2000; 29: 166-174.

30. Benowitz L, Yuqin Y. Optic Nerve Regeneration. Arch Ophthalmol 2010 Aug; 128(8):1059-1064.

\section{COLABORACIÓN}

Los autores agradecen la colaboración de Geraldine Santos, Carolina Huayta y Stephen Paucarcaja. 